\title{
Making Graphics Tangible
}

\author{
Carlo H. Séquin
}

EECS, Computer Science, University of California, Berkeley

\begin{abstract}
This is a write-up of material presented at keynote talks at Virtual Reality 2012 and at Shape Modeling International 2012. Producing tangible, physical output becomes a growing role for computer graphics due to the emergence of inexpensive rapid-prototyping machines and on-line fabrication services. While producing small models with a layered manufacturing process has become very easy, creating larger and more durable objects still requires a designer to address an expanded list of issues. In this article some of these issues are discussed based on experiences the author has gained while designing various physical artifacts ranging from mathematical visualization models and geometrical puzzles to large scale sculptures. Tangible artifacts also gain importance at the beginning of a design process and as input to computer graphics tools. An argument is made and an outline is given for new ways and better user interfaces to enter the geometry of inspirational artifacts into our virtual CAD environments.
\end{abstract}

Keywords: Procedural modeling, tangible modeling tools, rapid prototyping, durable physical output.

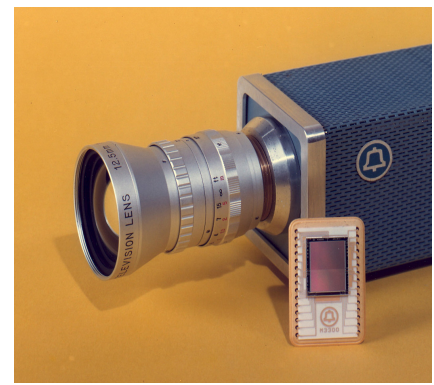

a

Figure 1: (a) CCD solid state image sensor; (b) Soda hall, U.C. Berkeley.

\section{Introduction}

2 For most of my life geometry and geometrical constructions ${ }_{3}$ have been my profession as well as my hobby. In some in4 stances, the realization of a particular design required an army 5 of people and a major organization for turning the design into 6 a usable physical artifact. My first job after graduation placed 7 me in the group at Bell Labs that had just invented Charge${ }_{8}$ Coupled Devices (CCD). Over the next few years I designed ๑ ever larger solid state image sensors, culminating in 1973 in 10 a CCD imaging chip with enough resolution to be compatible 11 with the American broadcast TV format (Fig.1a). The produc12 tion of three good chips to be placed around a color separation ${ }_{13}$ prism involved the fabrication of tens of silicon wavers, requir14 ing several dozen individual processing steps in a very sophis15 ticated fabrication line for integrated circuits. A decade later ${ }_{16}$ I was spearheading the design effort of Soda Hall, the home 17 of Computer Science on the Berkeley campus. There again, 18 the realization of that design took several architects, many con${ }_{19}$ tractors and subcontractors, and kept a large number of skilled

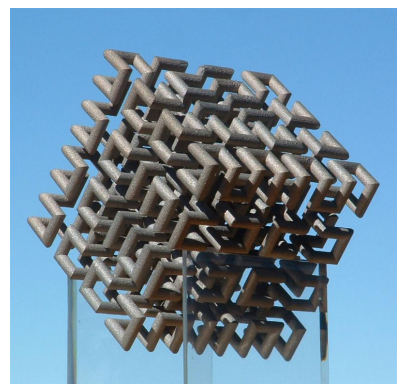

a

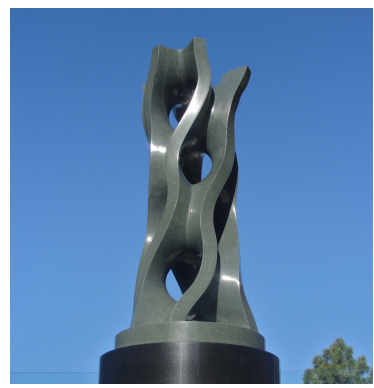

b
Figure 2: Large and/or complex physical entities that started out as a computer graphics description: (a) Hilbert Cube 512 ; (b) Pillar of Engineering .

20 workers busy for two years to produce the final building (Fig. 1b).

Fortunately, not all physical artifacts take that long from 22 initial concept to a first realization. Because of the emergence ${ }_{23}$ of rapid-prototyping (RP) machines and services, some smaller 24 objects can now be realized in a matter of hours or days. This 25 has had a tremendous impact on product design in many areas, ${ }_{26}$ ranging from car manufacturing to computers and to household ${ }_{27}$ items such as toasters, or sports articles such as running shoes. ${ }_{28}$ Most of these products now undergo a design cycle that in29 volves a few rapid prototyping models. The final product how30 ever, still requires a much more involved and more expensive 31 process, for instance the creation of sophisticated molds for in32 jection molding.

In the world of computer graphics, every year hundreds of ${ }_{34}$ stunning renderings and virtual artifacts with which users can 35 interact in video-game settings are being created with interac${ }_{36}$ tive modeling tools. Because of the emergence of affordable RP 37 machines, e.g. MakerBots [1] and readily available fabrication ${ }_{38}$ services such as Shapeways [2] or Ponoko [3], an ever grow- 


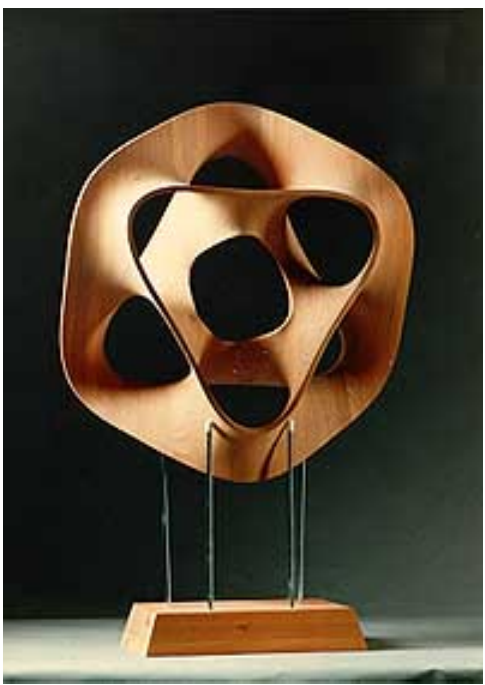

a

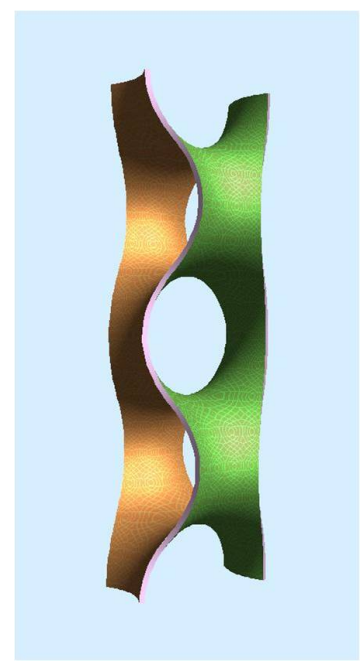

b

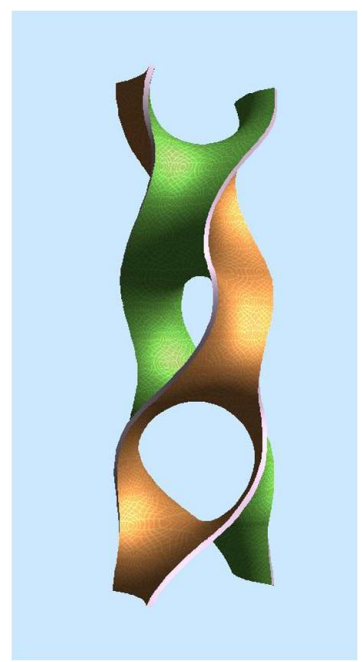

C

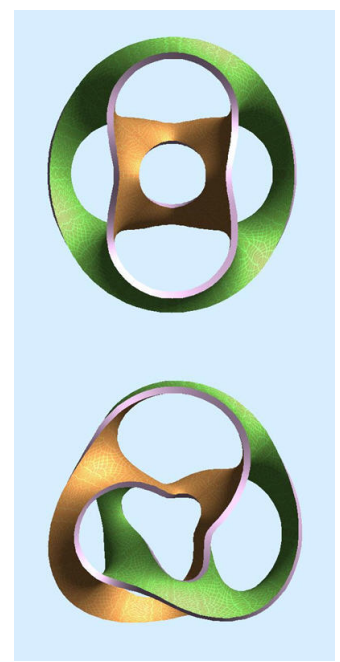

d

Figure 3: (a) Collins' Hyperbolic Hexagon ; (b) Scherk tower; (c) twisted Scherk tower; (d) corresponding Scherk tower loops.

${ }_{39}$ ing fraction of these models may find their way into the world 40 of physical reality. People produce small replicas of their fa${ }_{41}$ vorite action figures, models of their designs in Second Life [4], 42 custom-made coffee mugs or cookie cutters, or pendants and ${ }_{43}$ ear rings. But still, to produce an object that serves a functional ${ }_{44}$ purpose in your car, in some exercise machine, or in a hand tool ${ }_{45}$ like an electric drill, requires a more substantial effort that takes 46 more than a single pass through the rapid-prototyping process. 47 In this paper I will describe what it takes to turn an ap48 pealing virtual design into a physical artifact in the context of 49 mathematical visualization models (Fig.2a), geometrical puz50 zles, and large and durable public sculptures that can be touched ${ }_{51}$ by spectators and can withstand the abuse by weather (Fig.2b). ${ }_{52}$ Section 2 gives the background story of how I got started with ${ }_{53}$ computer-aided modeling of large sculptures. Sections 3 and ${ }_{54} 4$ describe the realization of two large sculptures implemented 55 with different fabrication technologies. Section 5 discusses ad${ }_{56}$ ditional concerns that come into play when one plans to make 57 multiple copies of an artistic artifact such as an award trophy 58 to be handed to several recipients every year. Section 6 fo${ }_{59}$ cuses on making models on RP machines with the added con60 straint of models composed of multiple parts that must fit to${ }_{61}$ gether smoothly, such as dissection puzzles; it also addresses 62 approaches of reducing the fabrication costs of such models.

63 Sections 7 and 8 address design and user-interface issues, 64 to the extent that this front-end activity may involve tangible 65 physical artifacts. They conclude with a generalization of the 66 procedural modeling approach that I have used in most of my ${ }_{67}$ sculpture designs, called Inverse 3D Modeling. They end up 68 with a proposal of how to use tangible pieces of material as ${ }_{69}$ design building blocks in an immersive virtual work space.

Rapid prototyping technologies have advanced tremendously 71 in the last decade and have now become quite affordable. Sec72 tion 9 gives a brief preview of the possibilities that lie ahead in ${ }_{73}$ the near future.

\section{${ }_{74}$ 2. Scherk Collins Sculptures}

My interest in computer-assisted sculpture design started in 76 1995. It grew out of the need for a virtual prototyping tool 77 that would permit a quick evaluation of a variety of abstract ge78 ometrical forms to determine which ones of many conceptual 79 ideas would have the potential to be turned into a 3-dimensional 80 physical sculpture that would look interesting and aesthetically ${ }_{81}$ pleasing from most directions. This need arose after I had made 82 contact with sculptor Brent Collins [5], living in Gower, MO, 83 and we started to have weekly phone discussions in which we ${ }_{84}$ generated more conceptual ideas than could possibly be exam85 ined and evaluated with physical mock-up models.

In 1994 I had come across an article by George Francis [6] 87 in which he analyzed some of Brent Collins' wood sculptures ${ }_{88}$ from a topological and knot-theoretical point of view. One of ${ }_{89}$ the key elements in Collins' work is a composition of stacked 9o saddles and intertwined tunnels. I was intrigued by the sophis91 ticated way in which the edges of the various saddles merged 92 seamlessly into one another and by the fact that all this com${ }_{93}$ plicated geometry resulted in smooth and balanced surfaces. In 94 1995, after I had come across an image of the graceful Hyper95 bolic Heptagon (Fig.3a), I picked up the phone and contacted ${ }_{96}$ Brent Collins. Based on the analysis by George Francis, this 97 sculpture could be understood as a toroidal deformation of a ${ }_{98} 6$-story "Scherk tower." The latter is a finite cylindrical cut-out 99 (Fig.3b) from the center of Scherk's 2nd minimal surface [7].

100 Our first phone discussion started from the observation that 101 making a good 3D sculpture is not easy, if you want to make 102 it look good from all sides. Hyperbolic Hexagon shows some 103 weaknesses in this respect, because from some angles it dis104 plays some strange coincidences. This is because it has too 105 much symmetry: All six peripheral holes pass through the dom106 inant plane at $\pm 45^{\circ}$; and thus from certain viewing angles they 107 all close up simultaneously or show their full, open, circular 108 tunnels. 


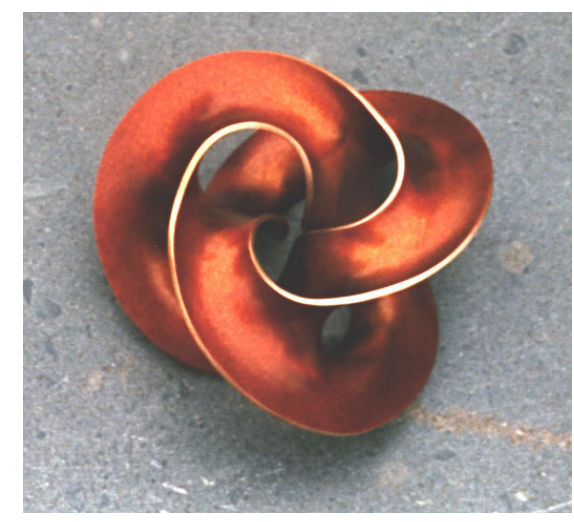

a

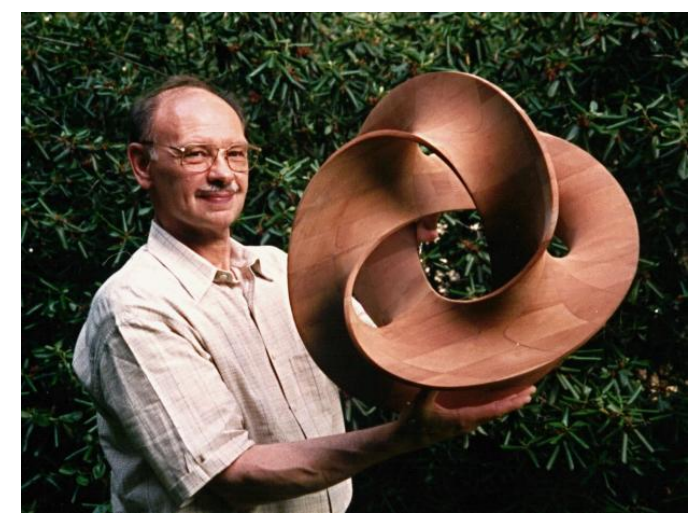

b

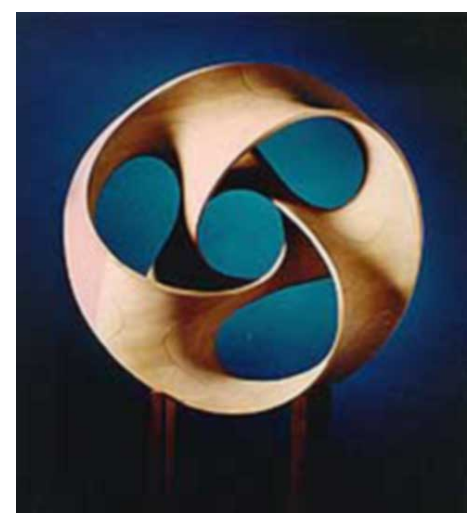

C

Figure 4: Hyperbolic trefoils: (a) Séquin's Minimal Trefoil ; (b,c) Collins' Trefoils with different azimuth angles.

We contemplated whether this could be fixed by adding 110 some twist into this structure, so that every hole would pass 111 through the dominant plane of the toroidal sweep path at a 112 somewhat different angle. Clearly such twist would have to ${ }_{113}$ be inserted in increments of $180^{\circ}$, so that the chain of saddles 114 would still close smoothly onto itself. We also realized that, if 115 there are an odd number of stories in this toroidal loop, an odd 116 multiple of $90^{\circ}$ twist is required to close the toroid smoothly; 117 but then the surface becomes single-sided, thus forming a non118 orientable Möbius configuration. Moreover, in the original $\mathrm{Hy}$ 119 perbolic Hexagon all four edges form their own separate, closed 120 loops. Adding twist to the Scherk tower interlinks the edges of ${ }_{121}$ this sculpture in interesting ways. For certain amounts of twist, 122 these edges will merge into one another after one pass around ${ }_{123}$ the twisted toroid, and it will then take several laps around the ${ }_{124}$ toroid to reach the starting point again. Analyzing the multi${ }_{125}$ ple intertwined torus knots that can be formed in such twisted 126 structures is rather intriguing.

${ }_{127}$ These insights were mathematically fascinating, but Collins 128 was interested in deriving new ideas for structures that would ${ }_{129}$ result in aesthetically pleasing physical sculptures. Typically, ${ }_{130}$ Collins first builds small scale models from PVC piping or em${ }_{131}$ broidery hoops, fleshed out with wire meshing and bees wax, 132 before he embarks on carving a large wood sculpture. Mak${ }_{133}$ ing a small scale model takes itself a couple of weeks. Thus ${ }_{134}$ it quickly became clear that Brent could not possibly keep up ${ }_{135}$ with making models for all the new concepts we discussed in ${ }_{136}$ order to judge their visual appeal.

${ }_{137} \quad$ At this point I decided that a computer-based visualization ${ }_{138}$ tool was our only hope of keeping up with our stream of ideas. ${ }_{139}$ The result was Sculpture Generator I [8], a special purpose 140 modeling program written in the $\mathrm{C}$ language, the geometry ker${ }_{141}$ nel of which comprised only about 3000 lines of code [9]. Within 142 a few months I had a first prototype running that allowed me to ${ }_{143}$ specify the number of stories in the loop, their combined total 144 twist, the azimuth orientation of the edges around the smaller 145 radius of the torus, as well as the thickness and extension of 146 the flanges, and various simulated surface properties (Fig.5). ${ }_{147}$ Now I could try out many different ideas and parameter com148 binations in a matter of minutes, and then focus on the most
149 promising configurations and optimize them for their aesthetic 150 appeal. Or I could investigate such questions as: What is the ${ }_{151}$ tightest toroid into which a Scherk tower with only three stories ${ }_{152}$ could be curled up. The latter question led to my version of the ${ }_{153}$ Minimal Trefoil (Fig.4a). I gave myself the constraint that the 154 tunnels would start out with a circular profile, trying to avoid 155 any vertical stretching of the 3-story Scherk tower before clos156 ing it into a loop. I succeeded in doing this for an azimuthal 157 orientation of $45^{\circ}$ of the three saddles in the ring. Collins also 158 hand-designed and built two trefoils with different azimuthal 159 orientations $\left(0^{\circ}\right.$ and $\left.45^{\circ}\right)$, but in both instances he stretched the 160 hole-saddle chain substantially, so that he would obtain a cen${ }_{161}$ tral hole that was comparable to the tunnels in the periphery. ${ }_{162} \mathrm{He}$ wanted to keep all of these tunnels large enough so that he ${ }_{163}$ could easily get one of his hand inside for the final carving and ${ }_{164}$ polishing (Fig.4b,c).

${ }_{165}$ One key technical issue I had to address in the development ${ }_{166}$ of Sculpture Generator I was the question how I should best ${ }_{167}$ represent the geometry of these shapes to maximize the bene${ }_{168}$ fit of the inherent modularity and symmetry in these toroidal ${ }_{169}$ structures. Even though these shapes were inspired by the min170 imal surfaces formed by soap films spanning some boundary 171 loop, they are not typically true minimal surfaces. For aesthetic 172 reasons, most of the twisting ribbons connecting adjacent sad${ }_{173}$ dles have a much stronger lateral curvature than their longitu174 dinal curvature. Thus I decided to model one quarter of a stan175 dard biped saddle with a stack of hyperbolas with ever more ${ }_{176}$ pointy hyperbolic curves that eventually end up as two straight 177 lines crossing at a $90^{\circ}$ angle at the saddle point (Fig.6a). Ad178 jacent hyperbolas are connected with triangle strips to form a 179 tessellated surface with an adjustable degree of approximation 180 (Fig.6b). This small piece of surface can then be deformed as ${ }_{181}$ needed for a particular sculpture. Properly rotated, mirrored, 182 and translated, it will form a complete 1-story saddle. The de183 sired number of saddles can then be stacked on top of one an184 other, and the whole assembly can be given a suitable amount 185 of twist before it is bent into a loop and closed into the final 186 toroid. If saddles of a higher order are desired, e.g., a 3-way ${ }_{187}$ "monkey saddle," the $90^{\circ}$ sector forming the fundamental do188 main of a basic biped saddle can be compressed into a wedge 


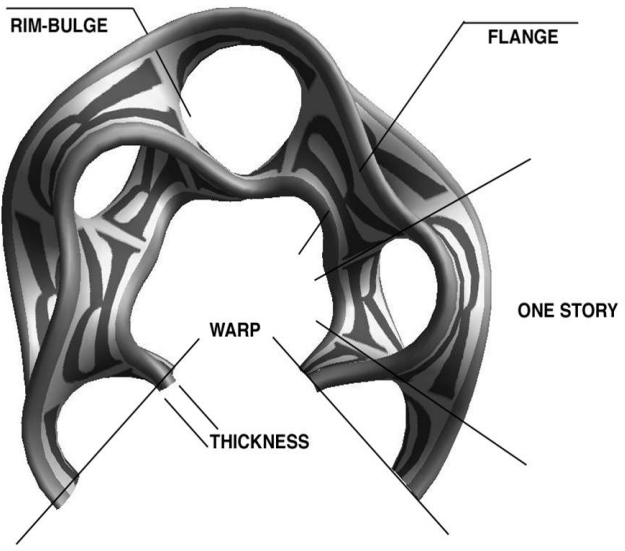

Figure 5: Design parameters in one story of the Scherk-Collins toroid.

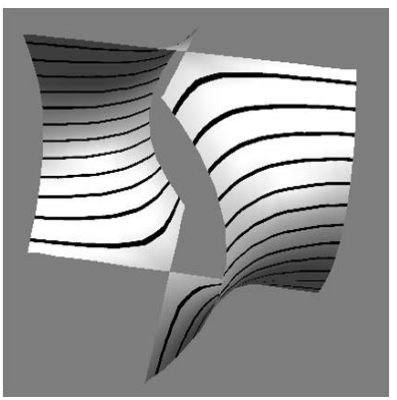

a

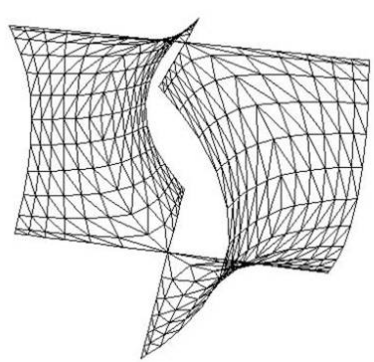

b
Figure 6: Internal shape representation: (a) set of stacked, parametrized hyperbolas, (b) approximated with symmetrical pairs of triangle strips.

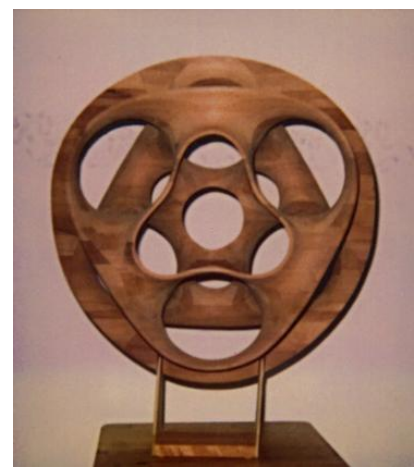

a

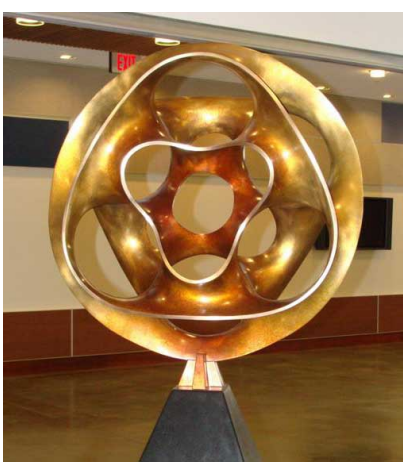

b
Figure 7: Hyperbolic Hexagon II : (a) 1997 wood master carved by Brent Collins; (b) 2009 bronze sculpture cast by Steve Reinmuth, installed in Sutardja Dai Hall at U.C. Berkeley.

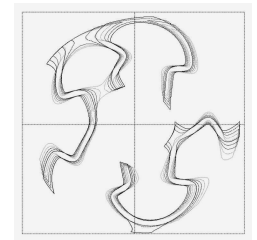

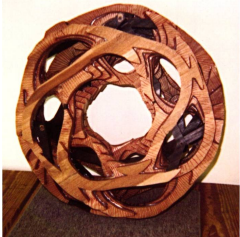

b

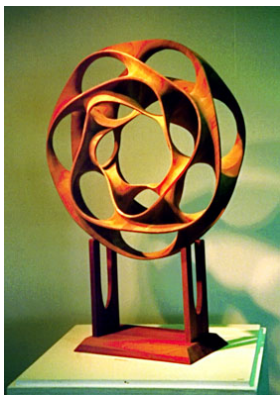

Figure 8: Heptoroid: (a) blueprint of one of its slices, (b) laminated assembly, (c) final sculpture.

189 with only a $60^{\circ}$ opening, and this wedge is then instantiated 2 190 six times around the z-axis. Compressing it into $180^{\circ} / n$ and in${ }_{191}$ stantiating it $n$ times in a rotationally symmetrical manner will 192 generate an $n$-th order saddle.

${ }_{193}$ By the end of 1996, Sculpture Generator I [8] had evolved 194 into a fairly robust program with several different output mod195 ules. Besides interactive virtual renderings on a computer screen, 196 the program also could output a boundary mesh of these solid 221 ${ }_{197}$ shapes in the .STL file format, which is accepted by most lay${ }_{198}$ ered manufacturing machines. I also added a program module 199 that could output full-size blue prints of cross sections spaced 200 at regular intervals, say $7 / 8$ of an inch apart, corresponding to 201 the thickness of the wooden boards from which Brent Collins 202 was planning to build such sculptures. Hyperbolic Hexagon ${ }_{203}$ II was the first large sculpture that Collins built from a set of 204 blue prints generated by Sculpture Generator I. He cut the in${ }_{205}$ dividual profiles from $7 / 8$ inch thick walnut boards, assembled 206 them with industrial strength glue, then fine-tuned the shape ${ }_{207}$ with hand tools, and honed the surface to perfection. Figure 2087 a shows our first truly collaborative piece. In 2008 this wood 209 sculpture was used as the master for making a silicon rubber 210 mold, and Reinmuth Bronze Studio [10] produced two bronze ${ }_{211}$ replicas. The second one was cast just in time for the opening of ${ }_{212}$ the CITRIS headquarter building on the U.C. Berkeley campus ${ }_{213}$ (Fig.7b).

Hyperbolic Hexagon II has enough symmetry that Collins 215 thinks he might have been able himself to generate the basic ge${ }_{216}$ ometry with ruler and compasses. On the other hand, he admits ${ }_{217}$ that he could never have conceived and drawn the geometry of 218 our second collaborative piece, the Heptoroid (Fig.8). This is a ${ }_{219} 7$-story structure with $4^{\text {th }}$-order saddles and a total twist of $135^{\circ}$ 220 (3/8 of a full turn). Figure 8a shows one of a dozen actual 3${ }_{221}$ foot wide blue prints, produced in my Sculpture Generator I. ${ }_{222}$ The five superposed traces show the geometry of this sculpture ${ }_{223}$ at top and bottom of the board, as well as at intermediate lev${ }_{224}$ els at $1 / 4,1 / 2$, and 3/4 of the board thickness. Again, Collins 225 used a saber saw to cut these shapes out of 7/8-inch thick wood 226 boards and laminated all the 12 profiles together with industrial${ }_{227}$ strength glue. In this way he obtained a first rough shape that 228 defines all the right symmetries, but shows strong stair-casing 229 on its surface (Fig.8b). He then grinds down the stair-casing 230 and creates a smooth, thinned-down surface, with one contin${ }_{231}$ uous rim that travels around the loop eight times before it re${ }_{232}$ turns to the starting point. The glue lines provide good cues for ${ }_{233}$ the smoothness of the shape - in addition to the haptic feed${ }_{234}$ back gained from running the hand over the surface. This is 235 a manual version of "layered manufacturing." Heptoroid was ${ }_{236}$ exhibited at Fermi Lab in 1998 (Fig.8c). Many physicists saw ${ }_{237}$ something that reminded them of their profession: a stellarator ${ }_{238}$ fusion chamber, or some structure relating to string theory. 


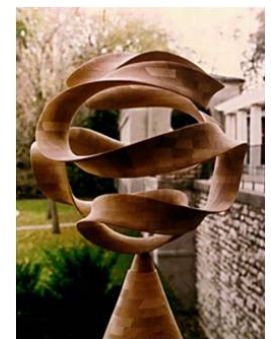

a

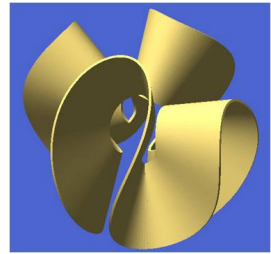

b

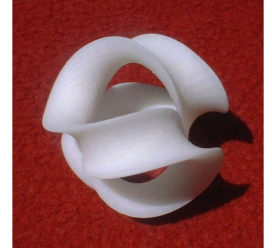

C
Figure 9: Sweeps along Gabo curves: (a) Collins' Pax Mundi wood master; (b) Gabo-4 ribbon; (c) Gabo-2 (baseball seam) sweep.

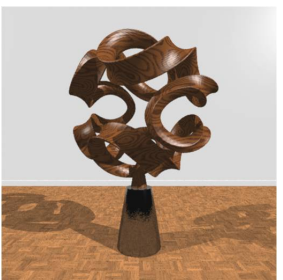

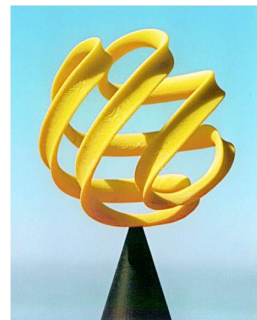

$\mathrm{b}$

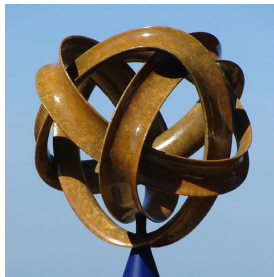

Figure 10: Viae Globi sculptures: (a) 5-lobe Gabo path; (b) Altamont sculpture; (c) Chinese Button Knot.

\section{3. Pax Mundi and Viae Globi}

240 The first commission to build a large metal sculpture con${ }_{241}$ cerned a different original model sculpted by Brent Collins. ${ }_{242}$ He created Pax Mundi completely independently shortly af243 ter we had started our collaboration (Fig.9a). It comprised a 244 highly curved ribbon undulating around the surface of a vir245 tual sphere. This new sculpture fascinated me as much as $\mathrm{Hy}$ 246 perbolic Hexagon, and I immediately set out to also capture it 247 in a procedural description. There was no way that Sculpture ${ }_{248}$ Generator I could emulate this geometry, because this sculp249 ture was based on quite a different geometrical paradigm. The 250 key module needed was some generalized sweep program that 251 could extrude an arbitrary cross section along a loopy sweep 252 path embedded in the surface of a sphere. The main question 253 was what might be the best way to parametrize the geometry 254 of this sweep path to be able to quickly generate a multitude 255 of different members of this sculpture family. The key associ256 ation came from some sculptures by Naum Gabo in which he 257 had wound up broad-rimmed annuli so that the outer rim would 258 roughly fall onto a sphere. So I introduced the concept of a 259 "Gabo curve," which is a path that undulates symmetrically up 260 and down around the equator of a globe. The key parameter is ${ }_{261}$ how many periods it takes to close the loop; in the case of Fig262 ure $9 \mathrm{~b}$ this number is 4 . Correspondingly, the seam on a base${ }_{263}$ ball or on a tennis ball is then a 2-period Gabo curve (Fig.9c). ${ }_{264}$ The geometry of the lobes can easily be represented by a B265 spline with just 3 parameters that define the amplitude, width, 266 and shape of each lobe. In this light, I could now describe Pax ${ }_{267}$ Mundi as an "amplitude-modulated 4-period Gabo curve."

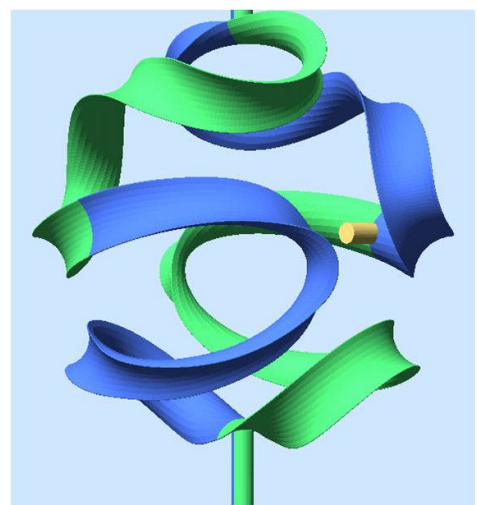

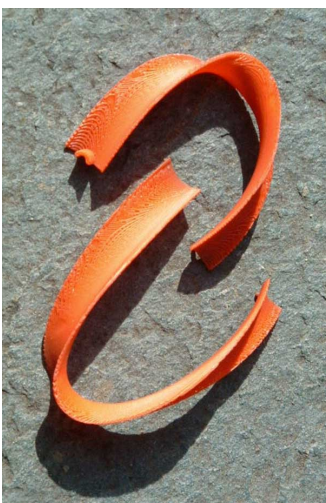

b
Figure 11: Segmentation of Pax Mundi: (a) Four replicas of the fundamental domain, (b) the domain split into two horseshoes.

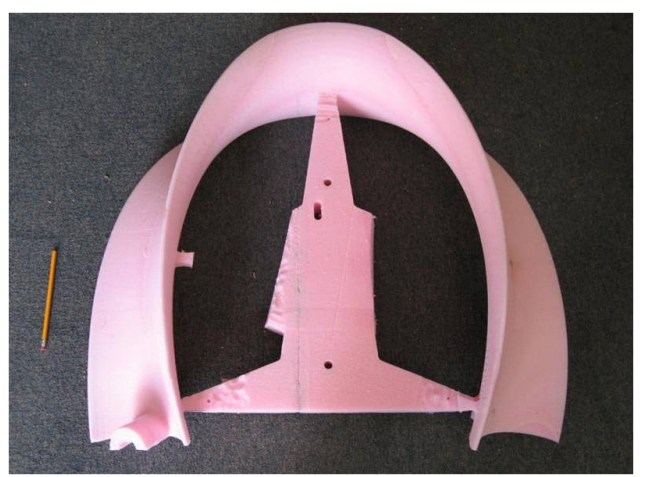

Figure 12: Small horseshoe produced on an NC milling machine.

269 veloped by several of my PhD students, I was now able to con270 struct a new and much more modular sculpture generator that 271 could capture this shape and produce many others like it. There 272 are three banks of slider controls. One defines the sweep path 273 on the sphere, another one the shape of the cross section of 274 the ribbon, and the third one the scaling and rotation of the 275 cross section during the sweep. By default the cross section 276 is kept in a fixed orientation with respect to the Frenet frame 277 of the sweep curve. Alternatively it can be propagated in a 278 rotation-minimizing manner along the sweep curve, with ad279 ditional control through a globally applied azimuthal rotation 280 of the cross section and an evenly spread out gradual twist; the 281 latter makes it possible to obtain an end-to-end alignment of 282 the cross section after a full pass around any arbitrary closed ${ }_{283} 3 \mathrm{D}$ space curve. Adding optional local twist parameters al284 lows the programmer to fine-tune the amount of twisting in 285 the neighborhood of critical loops or turns. This programming 286 environment proved to be particularly fertile and has led to 287 dozens of attractive designs (Fig.10a, 11a). Over the subse288 quent years I gradually broadened the concept of the Viae Globi 289 program and enhanced it to take more complicated undulations 290 (Fig.10b), to allow over/under crossings of the ribbon sweep291 ing along the sphere surface, and eventually leading to com292 pletely free-form space curves that could make intriguing knot293 ted structures (Fig.10c). 

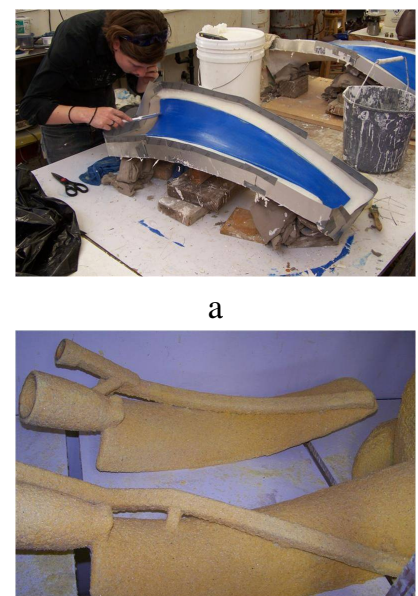

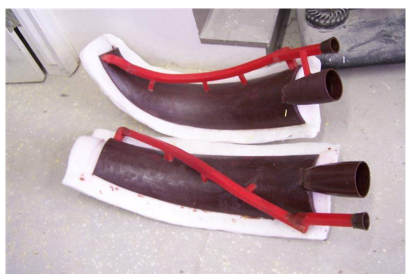

$\mathrm{b}$

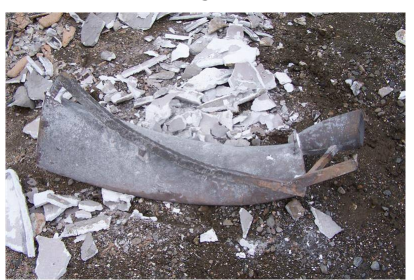

d
Figure 13: Realization of Pax Mundi in bronze: (a) coated part; (b) wax copy; (c) plaster shell; (d) freed up bronze.

294 In 2007 Collins obtained the commission to make a large 295 metal version of Pax Mundi for the H\&R Block headquarters in ${ }_{296}$ Kansas City. I used my program to generate the scaled-up ge297 ometry at the desired size ( 6 foot diameter), properly slimmed 298 down to fit within the weight limit ( $\leq 1500$ pounds) and budget 299 constraints $\left(\leq \$ 50{ }^{\prime} 000\right)$ while maximizing its aesthetic appeal. 300 Moreover, I had the responsibility to figure out the minimal set 301 of master modules that would have to be produced to create the 302 necessary molds in which segments of this sculpture could be 303 cast. The minimal amount of "master geometry" for this shape 304 turned out to be $1 / 4$ of the whole sculpture. I defined this geom305 etry as a curvy sweep through space (Fig.11a) and also provided 306 this segment with alignment tabs, so that the four pieces could 307 be joined with perfect alignment. Unfortunately the gantry of 308 the NC milling machine available to us had a clearance of only ${ }_{309} 14$ inches and could not handle my complicated, bulky 3D part. ${ }_{310}$ So I split the ribbon geometry into two smaller horseshoe pieces 311 as shown in Figure 11b, and provided additional alignment tabs. 312 Both halves are now relatively flat and fit under the gantry of ${ }_{313}$ that milling machine.

314 However both these horseshoes were still too large to fit 315 into the kiln in Steve Reinmuth's bronze foundry [10]. Thus ${ }_{316}$ Reinmuth cut the smaller part (Fig.12) in half, and the larger 317 one into three parts. Judiciously he made a suitable jig that 318 later allowed him to re-align the individually cast parts into the 319 required horseshoe shape, since those cut-up segments had no 320 alignment tabs. The five different styro-foam master-geometry 321 modules were coated with (blue) plastic paint (Fig.13a) to yield 322 a smooth surface, suitable for making silicon rubber molds. ${ }_{323}$ These molds are then used to cast four identical positive copies ${ }_{324}$ each in (brown) wax, to be used in a classical investment-casting 325 process: All wax parts are provided with the (red) sprues and 326 runners and funnels into which the molten bronze will be poured 327 (Fig.13b). This whole assembly then gets dipped repeatedly 328 into plaster slurry to make a ceramic shell (Fig.13c). These ${ }_{329}$ shells then get fired in a hot kiln. In this process, the wax runs ${ }_{330}$ out, leaving a cavity of the desired shape for the bronze. After

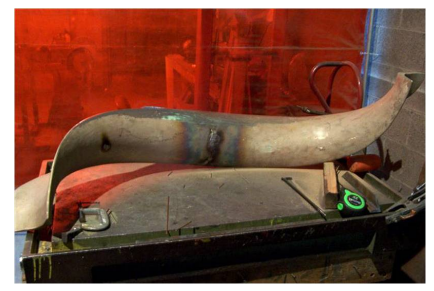

a

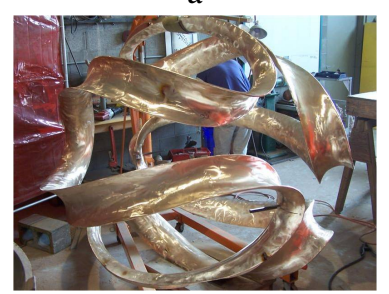

C

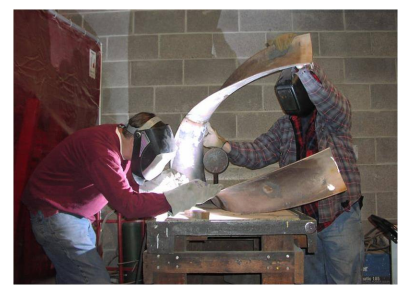

b

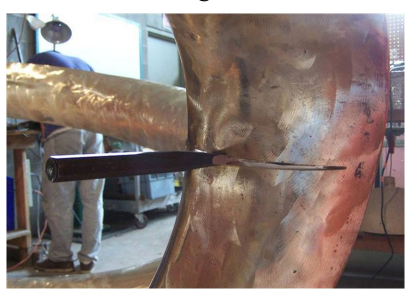

d
Figure 14: Pax Mundi assembly: (a) fitting together two segments, (b) assembling one horse shoe, (c) assembly completed, (d) cuts with wedges to elongate sculpture.

${ }_{331}$ the liquid bronze has been cast and has cooled down, the ce${ }_{332}$ ramic shell is smashed and removed (Fig.13d) - freeing up the ззз bronze part. On all 20 cast parts, the sprues and runners are 334 cut away, and a lot of cleaning and polishing has to be applied 335 before the pieces are ready for assembly (Fig.14a). First the ${ }_{336}$ eight horseshoes are re-assembled (Fig.14b), and then they are ${ }_{337}$ combined into the complete undulating ribbon (Fig.14c). All 338 the welds are ground down to a perfectly smooth surface.

But at this stage we had a bad surprise: The whole sculp340 ture sagged under its own weight by about $15 \%$ of its over${ }_{341}$ all height - and it was noticeably no longer spherical! Steve ${ }_{342}$ Reinmuth found a good solution, but it took several working ${ }_{343}$ days to fix the problem: He hung the sculpture from its highest ${ }_{344}$ point and let it stretch under its own weight. In addition he cut ${ }_{345}$ gaps half-way through the ribbon in four of the mostly horizon${ }_{346}$ tal hairpin curves, which enhanced the lengthening even more ${ }_{347}$ (Fig.14d). In this elongated state, those gaps were then filled 348 with bronze welds leading to a vertically stretched ellipsoidal 349 overall shape. When the complete sculpture was finally sup350 ported from its central point at the bottom, it settled back to an 351 almost perfectly spherical shape. The main lesson learned for 352 future ribbon sculptures of this kind is to do some basic stiffness 353 analysis, because physics is important too - not just geometry! 354 The assembled and polished shape is then subjected to a se${ }_{355}$ lect set of chemicals applied with a spray flask and the heat from 356 a hand-held flame torch (Fig.15a). With the skilled hands of an 357 experienced craftsman an even, and possibly smoothly varying 358 patina can be applied, which turns this geometrical form into a 359 true work of art. The installed sculpture is shown in Figure 15b. ${ }_{360}$ We think the result is highly successful - and it has indeed led 361 to additional commissions.

Right now a similar bronze ribbon sculpture is in the works 363 as a commission for a new science building for Missouri West${ }_{364}$ ern State University. It is based on Brent Collins' Music of the ${ }_{365}$ Spheres, another one of his original ribbon sculptures carved 366 in wood. The extended version of my Viae Globi program 


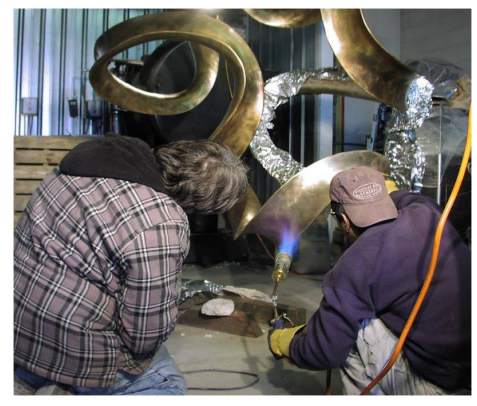

a

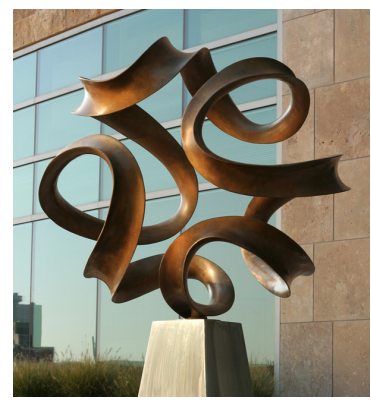

b

Figure 15: Pax Mundi installation: (a) applying patina; (b) finished sculpture.

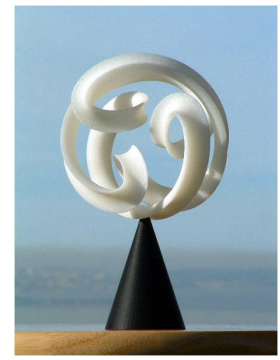

a

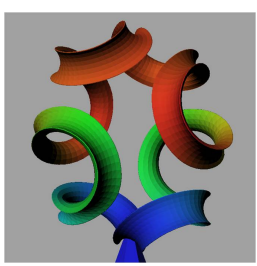

$\mathrm{b}$

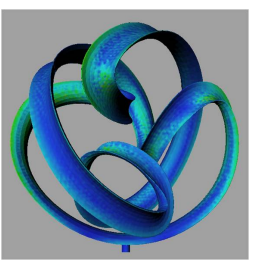

d

Figure 16: Music of the Spheres: (a) FDM maquette. Simulation analysis of gravitational deformation: (b) for Pax Mundi and (c) for Music of the Spheres .

${ }_{367}$ could easily capture this new shape and produce a maquette ${ }_{368}$ (Fig.16a)). Again, to make this into a 6 foot bronze sculpture, 369 the proportions had to be changed. Learning from my negli${ }_{370}$ gence with Pax Mundi, I subjected the computer model to some ${ }_{371}$ displacement analysis, using the ScanESSolve ${ }^{T M}$ for Rhino sim372 ulator [12]. The colors in Figures 16b,c indicate how much 373 each vertex moves from its design position under the influ374 ence of gravity. The red areas showing in the simulation of ${ }_{375}$ Pax Mundi indicate a 15\% displacement from the original po${ }_{376}$ sition (Fig.16b). The worst-case cyan color in the Music of 377 the Spheres simulation predicts a displacement of only about ${ }_{378}$ a 3\% (Fig.16c). Thanks to a different geometry and a some379 what thicker ribbon we should be much better off with this new ${ }_{380}$ sculpture. To be totally on the safe side, Reinmuth cast the up${ }_{381}$ per ribbon segments, which primarily act as pure loads, in the 382 form of hollow extrusions of the crescent profile.

\section{зsз 4. Millennium Arch}

384 Shortly after the large-scale realization of Pax Mundi, Collins 385 also obtained a commission for a large Scherk-Collins toroid. ${ }_{386}$ The design was coming directly out of Sculpture Generator I ${ }_{387}$ (Fig.17a). It was a 12-story ring with $4^{\text {th }}$-order saddles and $270^{\circ}$ 388 twist. This was the first large sculpture not based on an initial 389 model by Collins. This sculpture was destined to be hung in390 door under an atrium sky light in a community center in the ${ }_{391}$ City of Overland Park, Kansas. Thus it had to be light-weight 392 and somewhat translucent.

393 In 2007 David Lynn and Nova Blue Studio Arts, L.L.C., 394 in Seymour, MO, [13] helped us realize this 10-foot-diameter

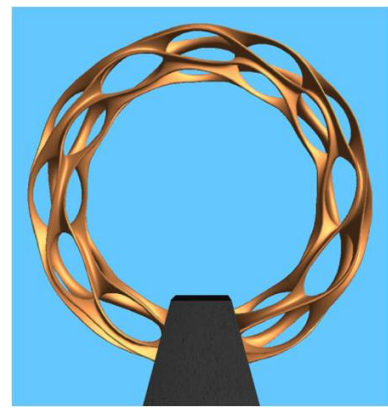

a

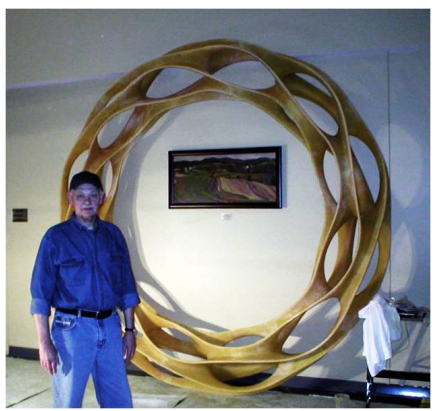

b
Figure 17: Millennium Arch: (a) computer model; (b) final sculpture.

395 sculpture (Fig.17b). The master geometry module was carved 396 out of high-density styro-foam on a large NC milling machine ${ }_{397}$ (Fig.18a). A mixture of glass-fiber and epoxy was employed to 398 form a reusable multi-piece shell around this module (Fig.18b). 399 Within that shell (Fig.18c) six copies were cast from a mixture 400 of polyester and glass fiber. Two half-circles of three modules ${ }_{401}$ each were then formed by fusing together some of the cast mod${ }_{402}$ ules. These arches were transported to their destination and as${ }_{403}$ sembled into a closed ring on the atrium floor (Fig.18d). The 404 completed ring (Fig.17b) was then hoisted into its final display 405 position.

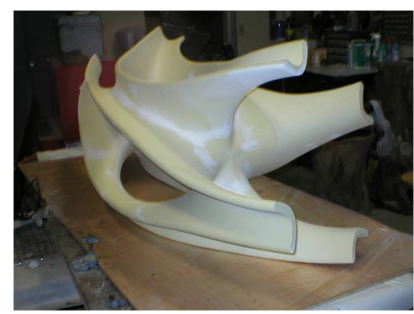

a

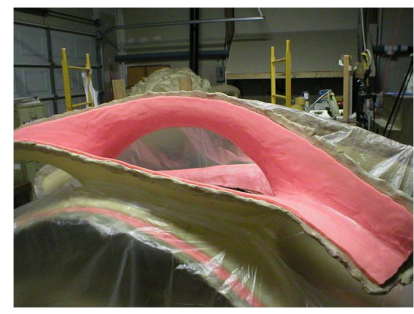

c

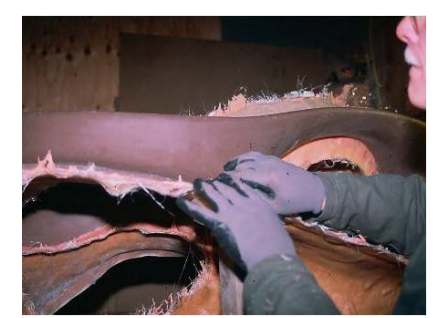

$\mathrm{b}$

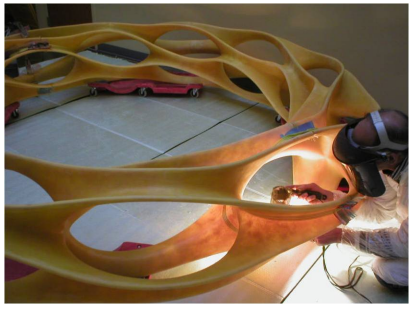

d
Figure 18: Realization of Millennium Arch: (a) NC-machined master geometry module; (b) fiberglass patches to form the mold; (c) complete mold of master module; (d) assembly of the arch. (Images courtesy of David Lynn, Nova Blue Studio Arts, L.L.C.)

\section{${ }_{406}$ 5. Making Multiple Copies}

407 Creating Pax Mundi and Millennium Arch were both one408 time, individual, custom realizations. The set of design con409 cerns expands if one wants to make multiple, perhaps even sev${ }_{410}$ eral dozen copies of a sculpture. And it expands even further if 411 a particular shape is supposed to be mass-produced - perhaps ${ }_{412}$ using production techniques such as injection molding. Here 


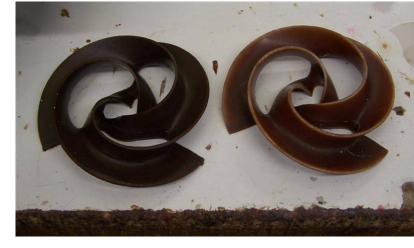

a

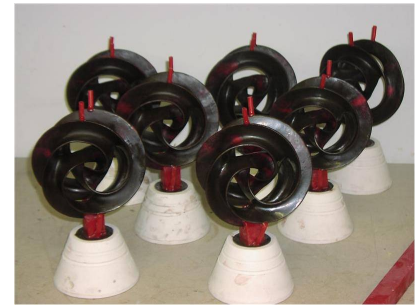

c

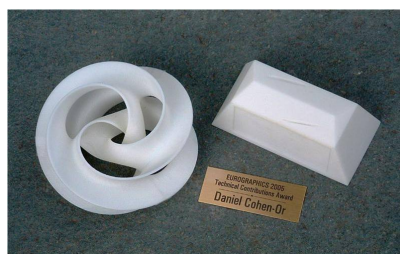

b

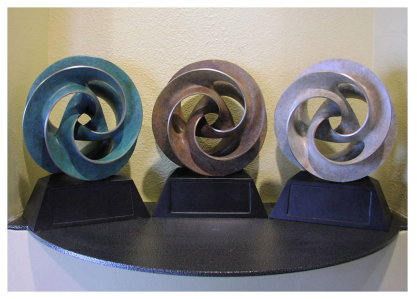

d
Figure 19: Eurographics Award: (a) master; (b) parts; (c) wax assemblies; (d) patinaed bronze awards.

${ }_{413}$ I will just offer a few observations based on my experience 414 with taking one of the Scherk-Collins motives and turning it 415 into an Award Trophy for EuroGraphics. Three or four of these 416 awards are being given out every year; thus overall I had to plan 417 for something that would be replicated several dozen times. In 418 this context, the molds should be relatively simple, robust, and 419 able to last for many dozen wax castings. The assembly of the 420 bronze sculptures should also involve as few pieces as possible 421 and require no tricky alignments.

422 The design that the EuroGraphics committee liked best was ${ }_{423}$ based on Whirled White Web, a twisted monkey-saddle trefoil ${ }_{424}$ from the family of Scherk-Collins toroids, which was carved 425 in snow at the 2003 annual Snowsculpting Championships in ${ }_{426}$ Breckenridge, CO [14]. Unfortunately this shape is too com${ }_{427}$ plicated to be made with a single mold and cast in one piece. ${ }_{428}$ It has a depth complexity of 3 or 4 in almost every direction ${ }_{429}$ and would require a very complicated multi-piece mold. But 430 it turns out that the shape can be split in its dominant central ${ }_{431}$ plane into two identical pieces; and these pieces only have a ${ }_{432}$ depth complexity of one or two measured perpendicularly to 433 this plane (Fig. 19a). A four-piece silicon rubber mold was able 434 to reproduce such a half-wheel element. Two of those elements ${ }_{435}$ could then be fused together and jointly be subjected to a stan${ }_{436}$ dard investment casting process (Fig.19c). Thus the complete ${ }_{437}$ trophy is assembled from only three metal parts: the wheel, 438 the base, and the individual engraved plaques (Fig.19b). Dif${ }_{439}$ ferent patinas distinguish the different award categories: The ${ }_{440}$ greenish-blue patina is for the Distinguished Career Award; the ${ }_{441}$ reddish-brown patina is for the Technical Achievement Award; ${ }_{442}$ the pale tan patina is for the Young Researcher Award (Fig.19d). ${ }_{443}$ Fabrication issues are as important as aesthetic concerns, 444 and the former have to be integrated into the design process 445 from a very early stage on. In the end, engineering concerns 446 often cause many more headaches than the artistic design con447 sideration.

\section{6. Dissection Puzzles and Other RP Models}

449 In the above example the final artifact, its material com450 position, and the manufacturing process had to be taken care${ }_{451}$ fully into account before the design of the trophy was finalized. 452 Another area, where material properties and tolerances play an ${ }_{453}$ important role is whenever several parts are designed that in 454 the end need to fit together snugly - perhaps to make a mov455 able joint or a snap-together junction between parts, such as 456 between a bottom and top half of the casing of a cell phone. ${ }_{457}$ A prime tutorial example is the construction of $3 \mathrm{D}$ dissection 458 puzzles, and this task domain was explored in a graduate course 459 on "Solid Modeling and Rapid Prototyping" at U.C.Berkeley in 460 the fall of 2011. Such puzzles are an excellent educational tool. ${ }_{461}$ They strongly emphasize 3D spatial thinking, and the fabrica462 tion gives hands-on feedback about accuracy and tolerances. ${ }_{463}$ Moreover, the resulting artifacts are fun to play with and to 464 show off to friends and relatives!

465 One of the assignments given to the students was: "Design a ${ }_{466} 2$ - or 3-piece geometrical puzzle in which a simple shape splits 467 into all congruent parts via a helical screw motion." All student 468 teams quickly figured out that the parting surfaces would have 469 to be one or more helicoids winding around a common straight 470 line, e.g. the z-axis. It was then their choice to select a suitable 471 overall shape, positioned symmetrically with respect to the cho472 sen system of helicoids, so that the resulting dissection (or tri473 section) pieces turn out to be congruent. Different teams picked 474 quite different shapes and different modeling approaches. The 475 teams that relied on the Berkeley SLIDE [11] software, which 476 offers very general, versatile sweep constructs, typically defined 477 a cross section (Fig.20a) and then swept that cross section along 478 a helical path (Fig.20b), while applying some uniform scaling 479 as a function of the z-value of the sweep path (Fig.20c). When 480 a teardrop scaling profile is used (Fig.20d), the puzzle shown in ${ }_{481}$ Figure 20e results.

482 Another team of students, who had access to SolidWorks 483 [15], which provides Boolean Constructive Solids Geometry ${ }_{484}$ (CSG) operations, decided to partition a cube along a space di485 agonal into three congruent pieces, which by themselves also 486 exhibited 2-fold rotational symmetry. To accomplish this, they ${ }_{487}$ adjusted the pitch of the helicoids so that each cutting surface 488 makes a $780^{\circ}$ turn around the $\mathrm{z}$-axis while sweeping from the 489 top to the bottom corner of the cube. Figures $21 \mathrm{a}, \mathrm{b}$ show two 490 different assembly stages of this puzzle. Figure $21 \mathrm{c}$ shows what 491 the individual parts looked like as they came out of the fused 492 deposition modeling (FDM) machine.

In all these puzzles, issues of geometrical design, numeri$494 \mathrm{cal}$ accuracy, and suitable tolerancing had to be addressed. For 495 some designs like the trisected "teardrop" - or "upside-down 496 ice-cream cone" (Fig.20e) the geometry was so tight and the 497 friction was so high that the three pieces could not be fully 498 screwed together until the helicoidal parting surfaces had been 499 sanded thoroughly. The designers of the trisected cube (Fig.21) ${ }_{500}$ struck a good compromise and hollowed-out the central por501 tion of the cube with a diameter equal to about half the cube 502 edge-length. This reduced friction dramatically, and the puzzle ${ }_{503}$ slipped together after only minimal sanding. The big question 


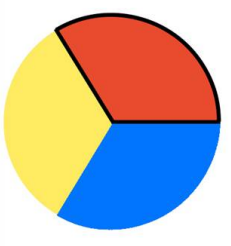

a

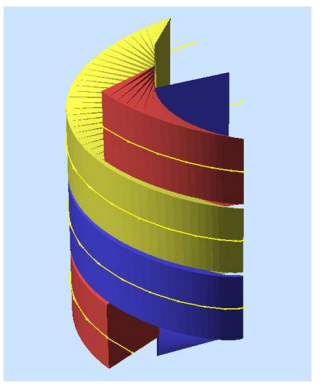

b

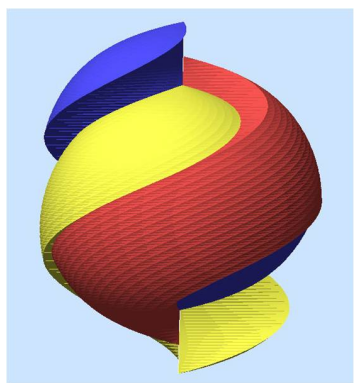

c

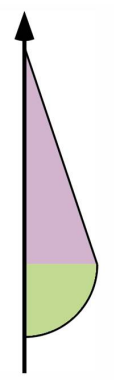

d

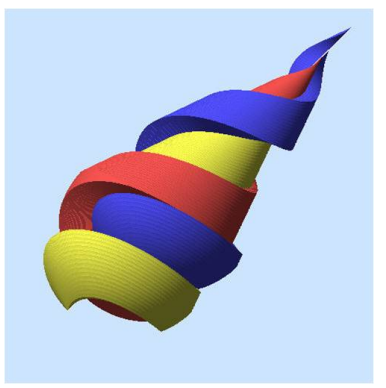

e

Figure 20: Helicoidal sweeps: (a) cross section; (b) simple sweep; (c) modulated scaling of cross section; (d) a different scale modulating function; (e) the resulting dissection puzzle geometry.

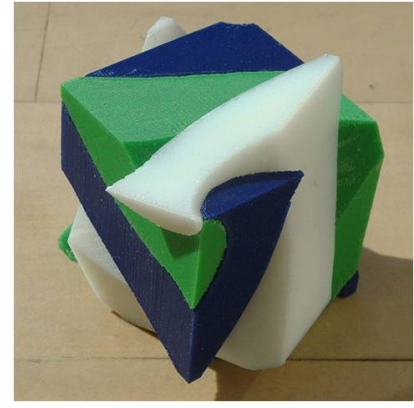

a

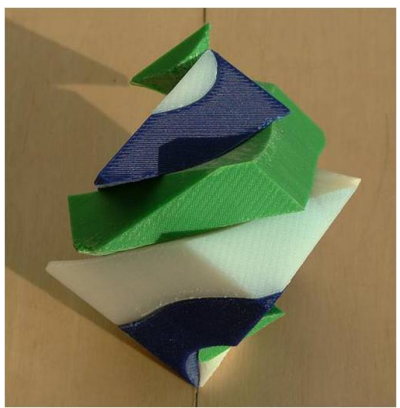

b

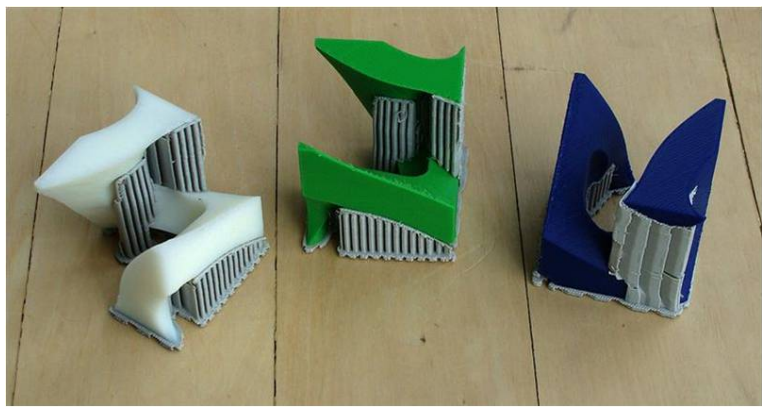

$\mathrm{c}$

Figure 21: Helical cube dissection: (a),(b) two different states of assembly; (c) the individual parts coming off the FDM machine.

504 is: How do you know what the right amount of clearance should 505 be? There were some puzzles that did not hold together well 506 and fell apart under the influence of gravity alone. My expe507 rience is that one cannot avoid doing an initial test-run on the 508 machine that one plans to use, submitting two or three design 509 variations that bracket one's best estimate of the proper amount 510 of clearance.

511 In this course we also addressed the question of how to min512 imize the costs of making parts on such a layered manufacturing 513 machine. To first order, the cost of such a part is proportional 514 to the material used in making the part. As a dramatic example, 515 I showed the class a neat little burr puzzle that I had purchased 516 from Shapeways [2] for about \$10.- (Fig.22), and then I asked 517 the students: "How could I obtain the same puzzle scaled-up by 518 a factor of 10 without having to pay $\$ 10$ '000.- ?" Specifically I 519 asked them to come up with a design that would minimize the 520 material usage on our old FDM machine, a type "1650" from ${ }_{521}$ Stratasys [16].

${ }_{522}$ In our discussions we quickly came to the conclusion that ${ }_{523}$ it would be advantageous to build just the outline of the puz524 zle pieces, keeping the interior mostly hollow. However, since 525 the FDM machine builds some kind of scaffolding underneath 526 bridges and overhangs in the part geometry, building a com${ }_{527}$ pletely hollow cube is not possible, and the internal scaffolding ${ }_{528}$ can still use up a lot of build material. A reasonable approach ${ }_{529}$ is to decompose each puzzle piece into its constituent cubelets, 530 and then realize each such cubelet as a thickened edge-frame 531 (Fig.23a) - akin to a style used by Leonardo DaVinci to depict 532 some to the regular and semi-regular polyhedra. This approach

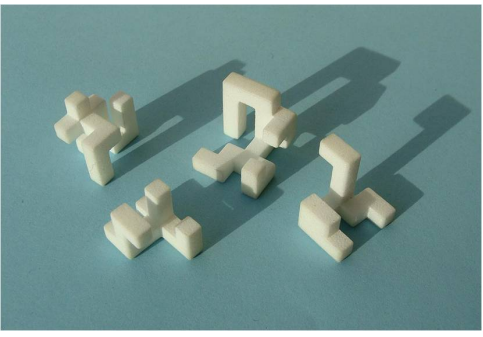

a

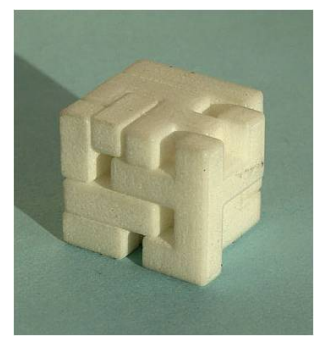

b
Figure 22: Small burr puzzle: (a) individual parts, (b) assembled puzzle.

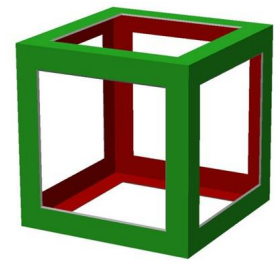

a
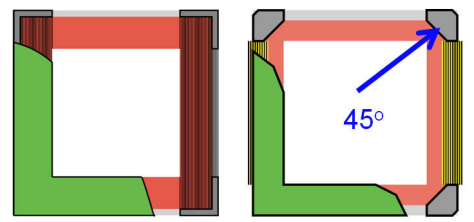

b
Figure 23: Cubelet frame: (a) Leonardo rendering, (b) cross sections through frame, without and with 45-degree bevel. 


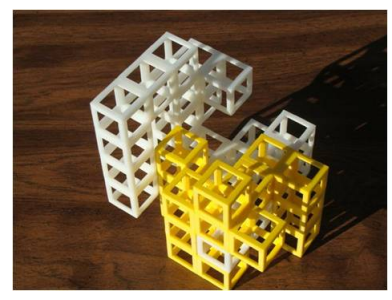

a

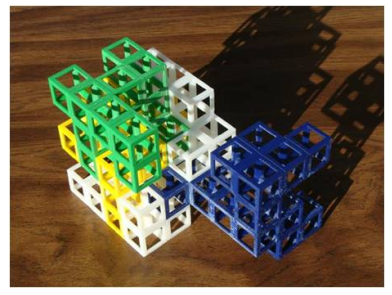

c

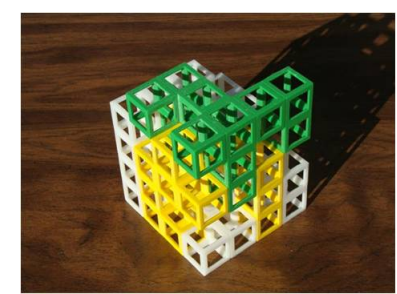

b

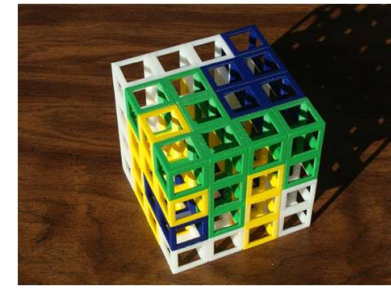

$\mathrm{d}$
Figure 24: "Framed" cubic burr puzzle in various stages of assembly.

${ }_{533}$ can save build material as well as support material, if the central ${ }_{534}$ portions of the cubelets can be kept free of any type of scaffold${ }_{535} \mathrm{ing}$, and if the support material can be restricted to the vertical ${ }_{536}$ "windows" in the sidewalls of every cubelet (Fig.23b).

${ }_{537}$ When trying to limit the scaffolding material to the vertical 538 walls, we had to contend with some idiosyncrasies of Quick${ }_{539}$ Slice, the software that comes with the Stratasys FDM machine ${ }_{540} 1650$. It slices the geometrical part into $10 \mathrm{mil}(0.25 \mathrm{~mm})$ thick ${ }_{541}$ layers and then drives the FDM machine to "paint-in" each 542 such layer with a back-and-forth motion (in the x-y-plane) of ${ }_{543}$ the nozzle that dispenses the hot, semi-liquid ABS plastic. In ${ }_{544}$ this program, the user has the option to specify what kind of ${ }_{545}$ supports the machine is supposed to build. First we tell the ma${ }_{546}$ chine to simply build straight, vertical support structures. Since 547 we expect all support structures to be small and locally confined 548 to the vertical walls of the cubelets, there is no need to use any ${ }_{549}$ tapered lateral growth for stability. Furthermore we specify that 550 overhanging faces of $45^{\circ}$ or steeper need no support structures, ${ }_{551}$ but can be built by relying on cantilevering outwards the beads 552 in subsequent layers by half their diameter. Moreover, we in553 ternally bevel the cube frame at a $45^{\circ}$ angle and thereby limit 554 the scaffolding to be constructed to a thin support slab inside 555 each vertical window. Figure $23 \mathrm{~b}$ shows a schematic cross sec556 tion through one cubelet with and without the $45^{\circ}$ beveling of 557 the frame; the green color represents the outer skin of the front 558 face; pink is the inside of the back face; the cross section of 559 the frame is shown in gray; and the thin, black, vertical lines 560 depict the area of space filled with support material. In this way ${ }_{561}$ the material costs can be reduced by about a factor 100 over a 562 simple, 10-fold scaled-up version of the original solid model. ${ }_{563}$ Figure 24 shows the complete scaled-up, "framed" burr puzzle 564 in various stages of assembly.

565 Clearly, in the design of a puzzle viable for mass produc566 tion, the envisioned manufacturing technique needs to be con${ }_{567}$ sidered right from the beginning. Such puzzles then become 568 a good exercise in design for manufacturing (CAM). Professor ${ }_{569}$ Paul Wright in Mechanical Engineering and I have been run-

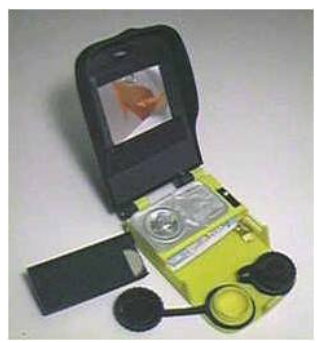

a

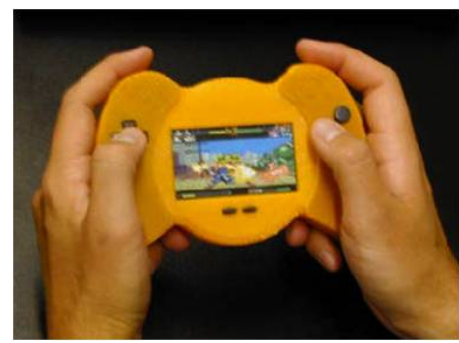

$\mathrm{b}$
Figure 25: Product prototypes build with layered manufacturing: (a) maintenance kit for contact lenses; (b) hand-held video game controller mock-up.

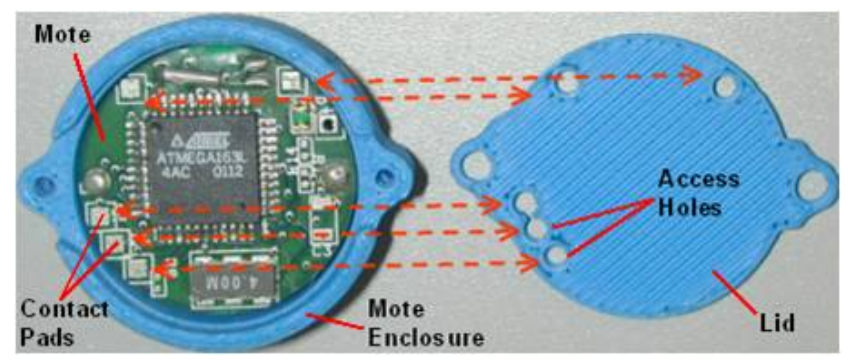

Figure 26: Multi-material prototype: A self-contained sensor node.

570 ning courses at U.C. Berkeley that also focus on the design for 571 manufacturing of more complex products that go beyond pure 572 shape design. Figure 25 shows two models that students have 573 built in this context: a compact travel kit for the maintenance 574 of your contact lenses (Fig.25a) and one of several shapes pro575 duced in a study to test the tactile quality for hand-held video 576 game controllers (Fig.25b).

New problems that arise when one has to deal with a prod578 uct design in which several technologies and knowledge do579 mains need to be brought together and interfaced with one an580 other. An example is the self-contained "mote" shown in Fig${ }_{581}$ ure 26. The contact pads on the printed circuit board need to be 582 brought into alignment with the access holes in the plastic lid 583 of this self-contained sensor / transmitter / networking node.

\section{${ }_{584}$ 7. Capturing the Geometry of an Inspirational Model}

585 Now I want to address the issue of how to get started with 586 the design of one of these artifacts discussed above. It turns 587 out that in this context tangible objects also can (and should) 588 play a more important role. Often a design or re-design task 589 starts from some related inspirational artifact. Many of my early 590 abstract geometrical sculptures were inspired by an individual 591 piece of art work sculpted by Brent Collins (Section 2). With ${ }_{592}$ Sculpture Generator I [9] I custom-built a program to capture 593 the essence of Hyperbolic Hexagon. With the more general, 594 modular Viae Globi program in SLIDE [11], I extracted the 595 essence of Pax Mundi and of other ribbon sculptures (Section 596 3). In 2009 I started an effort with James Andrews to general597 ize this approach even more [17]. Our prototypical Interactive 598 Inverse 3D Modeling system starts from an existing artifact that 599 may only exist in some low-level, unstructured representation, 


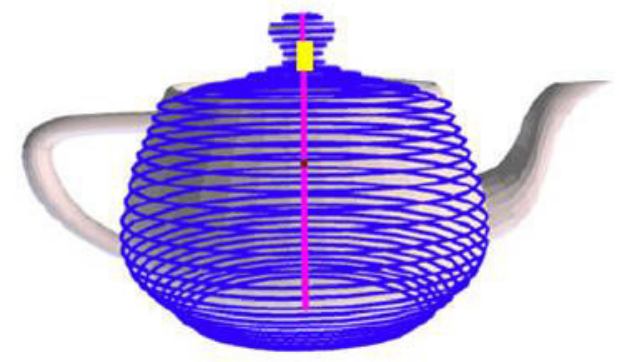

a

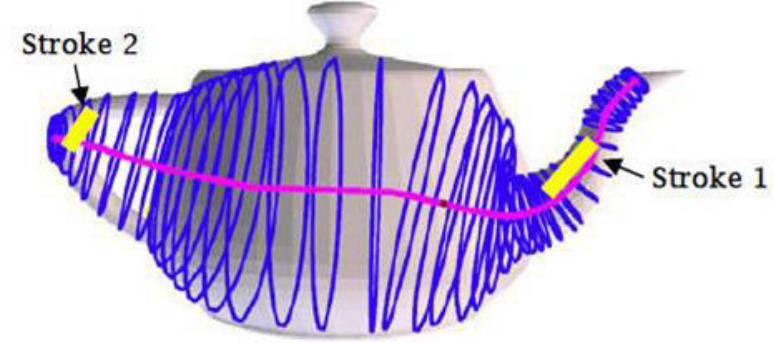

b

Figure 27: The shape of the Utah teapot interpreted in two different ways: (a) as a rotational sweep, (b) as a progressive sweep from the spout to the handle.

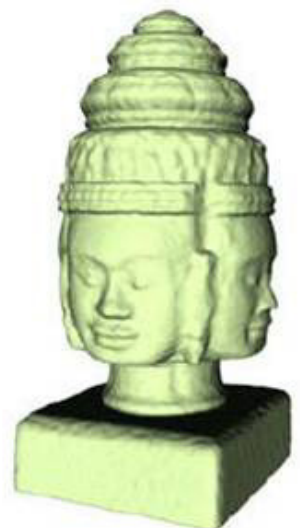

a

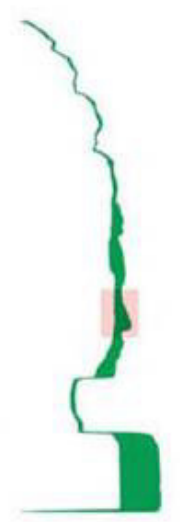

b

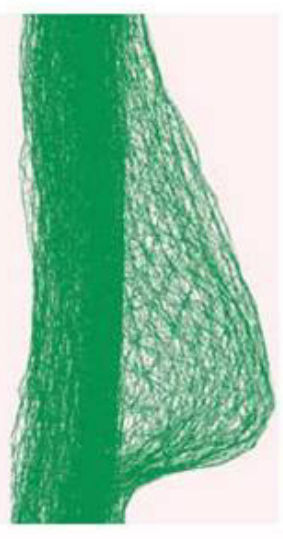

c

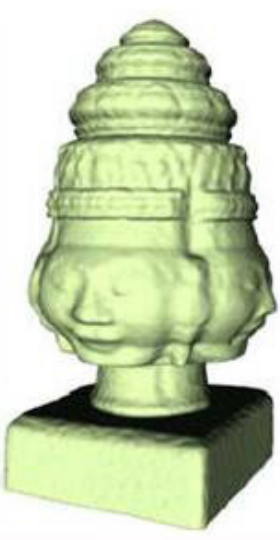

d

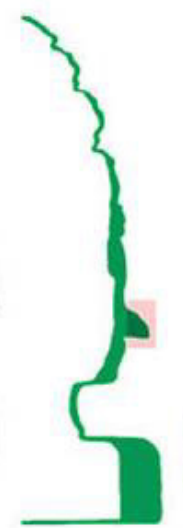

e

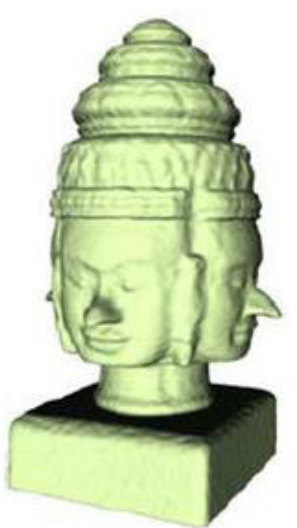

f

Figure 28: A statue with four-fold symmetry coerced into the framework of a rotational sweep: (a) the original statue, (b) its mesh rotationally collapsed into a thick "profile", (c) the area around the nose selected for editing, (d) the resulting fattening of the whole face region when the selected area is move outwards, (e) an alternative smaller area selecting only the nose tip, (f) the result when this smaller region is pulled outward.

600 such as a triangle mesh, a point cloud, or a set of images from ${ }_{601}$ different directions. With some user guidance the system then 602 extracts a high-level, parametrized, procedural description of ${ }_{603}$ this shape in a form that is most useful for the user in any plans 604 for adapting or redesigning it. Bringing physical artifacts into 605 the virtual world of a CAD environment can be quite labor in606 tensive and error prone, but we hope that this system will make 607 this process much more amenable.

608 The user loads the unstructured part description into the 609 computer and then selects on the surface of the virtual dis${ }_{610}$ play one feature or one basic shape at a time, while choosing ${ }_{611}$ the most desirable CAD representation for that segment. The ${ }_{612}$ belly of the famous Utah teapot may be extracted as a rota${ }_{613}$ tional sweep (Fig.27a) and its spout and handle as progressive ${ }_{614}$ sweeps with slowly varying cross sections. Alternatively the 615 system could also extract the whole teapot as a single progres${ }_{616}$ sive sweep from spout to handle (Fig.27b) - but this would 617 probably make much less sense for any reasonable re-design 618 task.

${ }_{619}$ Other objects may be decomposed into simple CSG primi620 tives (half-spaces, cubes, or cylinders) or pieces of quadric sur${ }_{621}$ faces and tori. These structures can then be modified easily by 622 using the parameters of the various extracted segments as han${ }_{623}$ dles, perhaps changing the sweep path or the cross section of a
624 progressive sweep or by moving some of the CSG primitives to 625 slightly different locations.

626 Here is a brief review of two of the parametrized extrac627 tion modules that we have implemented so far. First there is 628 the powerful sweep extractor mentioned above: With a first 629 yellow stroke (Fig.27) the user defines the start of a progres${ }_{630}$ sive sweep. The program cuts through the local geometry with ${ }_{631}$ a plane that is roughly perpendicular to the user-drawn stroke 632 and determines a first tentative cross section. This profile is 633 then swept in the direction of the user stroke, and the profile 634 shape as well as the sweep direction are fine-tuned, so that the 635 generated extruded surface element is as close to the input data 636 as possible. Once a first best-fitting segment has been estab${ }_{637}$ lished, the sweep is continued at both ends in roughly the same ${ }_{638}$ direction as the current segment, with possible small correc639 tions to the sweep direction and the orientation and scale of the ${ }_{640}$ profile. This process is iteratively repeated at both ends as long ${ }_{641}$ as the constructed surface does not deviate by more than some 642 specified error tolerance from the given input geometry. If the 643 process does not cover quite as much of some generalized cylin644 der geometry as the user would like, an additional stroke can be ${ }_{645}$ painted on some additional surface area yet to be covered, and ${ }_{646}$ the internal error tolerance will be suitably increased, so that ${ }_{647}$ the sweep extraction process can continue as far as the drawn 


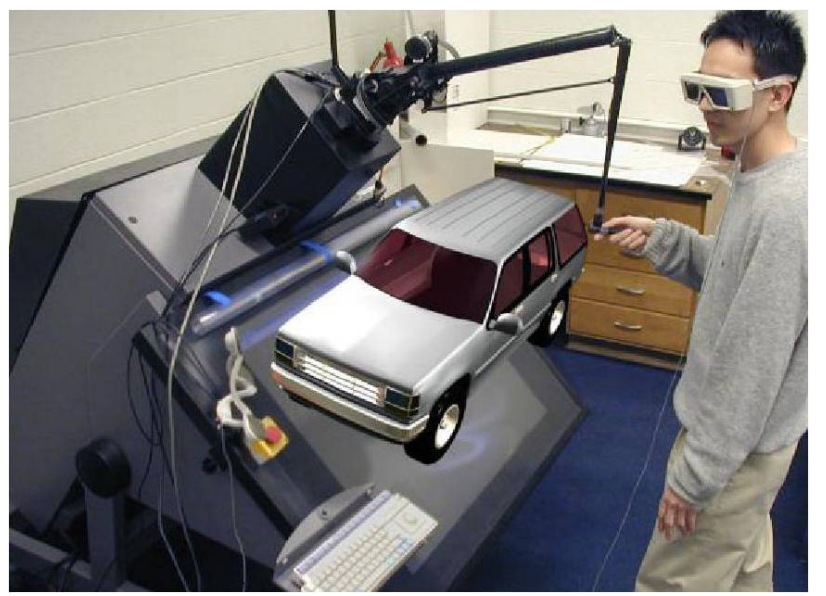

Figure 29: Virtual work space with haptic feedback

648 stroke indicates (Fig.27b). Conversely, with a different type of 649 stroke, the extent of the sweep extraction can be restricted. Dif650 ferent starting strokes and different error tolerances result in a 651 wide variety of possible extracted sweeps. The selected sweep ${ }_{652}$ paths and profiles can then be edited independently at interac${ }_{653}$ tive speeds.

${ }_{654}$ A different extraction module tries to fit a generalized ro655 tational sweep to the surface areas selected by the user. This 656 module cannot only match surfaces with strict rotational sym657 metry but can also extract helical and spiral sweeps [18] [19]. 658 In all our extraction modules, the reconstructed, parametrized 659 surface can either be used directly as the starting point for a 660 new design, or the surface details of the input model can be ${ }_{661}$ preserved and reused. To allow this, the difference between the ${ }_{662}$ actual input geometry and the extracted surface is stored as a 663 displacement map, which can be re-applied to any edited ver${ }_{664}$ sion of the reconstructed surface. This is demonstrated in Fig665 ure 28 with a roughly cylindrical statue. The whole shape is first 666 approximated by a rotational sweep, and all the vertices as well ${ }_{667}$ as their interconnecting edges are then rotationally collapsed 668 into the green profile bundle shown in Figure 28b. Portions 669 of this bundle can now be selected and modified interactively 670 with respect to their distance from (and position along) the $\mathrm{z}$ 671 axis. If we select the whole region around the nose (Fig.28c) 672 and move it to the right, all the nose and cheek areas will be ${ }_{673}$ puffed up (Fig.28d). If, on the other hand, we only select the 674 tip of the nose (Fig.28e), only those vertices will change their 675 radial component, and we simply obtain four stretched noses 676 (Fig.28f). The variety of the extraction modules, and the versa677 tility with which they can be applied and the extracted geometry 678 can be used and transformed, make this a powerful way to get 679 a new design started, - if an artifact close enough to the envi680 sioned design can be found.

\section{${ }_{681}$ 8. Capturing an Initial Inspiration Existing in Your Mind}

682 But sometimes a model close enough to the intended de${ }_{683}$ sign may not exist, and the design is just a vision in one's mind. ${ }_{684}$ How do we get this into the computer? It would be nice if we

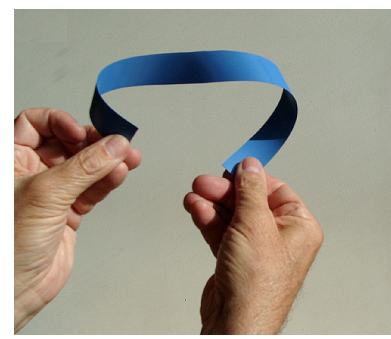

a

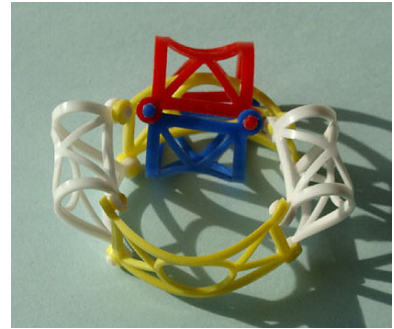

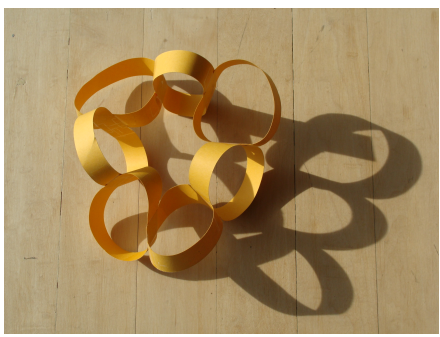

$\mathrm{b}$

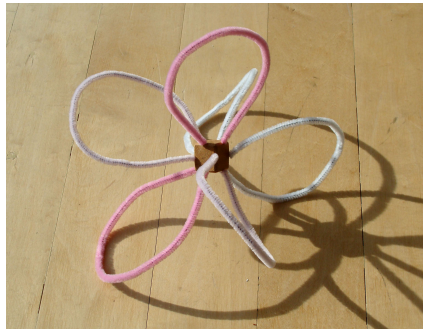

Figure 30: Shape elements to be inserted: (a) physical spline; (b) paper shapes; (c) plastic parts; (d) pipe cleaners.

685 could wave our hands and describe shapes in this way. Sev686 eral such sculpting systems have been presented over the last 687 two decades, e.g. [20]. A few years ago together with Profes688 sor Sara McMains in Mechanical Engineering, we built such 689 an interactive shape editor in an immersive 3D environment 690 [21]. Through a head-tracked pair of shutter glasses, the user 691 was seeing a 3D rendering of the design object in front of a 692 rear-projected display wall. With a haptic stylus attached to 693 a position-sensing articulating arm the user could interact with 694 that object. Vertices could be moved around, or the model could 695 be annotated or painted (Fig.29). However, the project was not 696 a smashing success! The tactile feeling was not solid; it was 697 more like drawing on a balloon with a ball point pen. Also, the 698 whole set-up was tedious and tiring. I found it impossible to 699 create conceptually new sculpture models in this environment. 700 Looking back over the last two decades of my own involve701 ment with sculpting and model making, I find that most novel 702 ideas start with something tangible in my hands. The things 703 that I am forming and combining are: wire, paper, scotch-tape, 704 paper clips, styro foam, clay, etc. Touch and proprioception 705 (knowing where your hands are even when blind-folded) play 706 an important role. Another important aspect of tangible objects 707 is that the materials properties contribute an important part of 708 the shape formation process. Bending a plastic strip or a thin 709 steel band creates nicely behaved spline curves. A knot made 710 from thick plastic tubing can be pulled tight mechanically to 711 produce some tightest configuration, - a process that would be 712 rather complicated to program in virtual space. Even famous 713 architects like Frank Gehry [22] occasionally use heavy cloth 714 or velvet to outline the roof of a new concert hall or museum.

715 Thus, what I really would like to do is to use whatever ma716 terial is most appropriate to form components of my design and 717 then integrate them with what is already there. One way in 718 which this could be accomplished is to work in an immersive 
719 virtual environment (but comfortably sitting down) in which I 720 can hold up various elements of the types shown in Figure 30, ${ }_{721}$ positioning and aligning them visually with the emerging com722 posite, and then, by tapping my foot (or clicking my tongue) 723 send a command to capture this proffered shape and add it into 724 the design description. This capture could be accomplished 725 with a fast 3D scanner, a structured light system [23], or a future 726 version of something like the Kinect [24]. The captured shape 727 would be presented to the designer through the Interactive In728 verse $3 D$ Modeling system described above (Section 7 ), so that 729 it can be represented with the most beneficial parametrization 730 for further editing, refinement, and optimization.

${ }_{731}$ An environment like this could offer the best of both the ${ }_{732}$ physical world and the virtual domain of computer graphics. It ${ }_{733}$ should allow us to readily grab a wide variety of tangible things, 734 or unstructured geometry descriptions, - whatever serves as a 735 possible inspiration or take-off point for our designs. How${ }_{736}$ ever, once such elements have entered the CAD design envi${ }_{737}$ ronment, these pieces of geometry can take on a new identity 738 in the form of materials that mimic the best of: clay, wire, pa${ }_{739}$ per, scotch-tape, styro foam, etc, but without the adversity of: 740 messy glue, gravity, or strength limitations; and they can repre${ }_{741}$ sent ideal pseudo-physical materials that bend as nicely as steel 742 wire, or stretch like a nylon hose, but are as strong as titanium, 743 and as transparent as quartz.

\section{9. Possibilities of Emerging RP Technologies}

745 Rapid prototyping technologies are currently in a phase of 746 fast development. Every year several new models of "Maker${ }_{747}$ Bots" and other layered fabrication machines are introduced 748 [25]. Many companies, as well as individuals, are experiment749 ing with new materials, different binders and support structures, 750 and are exploring new application domains for these emerging 751 technologies.

752 The large annual graphics conferences recently have de753 voted whole sessions to the topic of rapid prototyping and to 754 novel ways of producing physical, tangible output from com755 puter graphics designs. Some of these applications are quite 756 different from the traditional task of producing a physical 3D 757 object from a virtual model developed with one of the commer758 cial graphics or CAD programs. For instance, the development 759 of a bas relief of some arbitrary 3D scene [26] requires sophisti760 cated depth compression schemes, which are similar in their ap${ }_{761}$ proach to techniques used to compress high dynamic range im762 ages into renderings that can be displayed optimally on standard 763 graphics screens with limited dynamic range [27]. Other work 764 explores ways to make bas reliefs structured as height fields at 765 the pixel level to generate physical panels that will produce one 766 or possibly several images when illuminated at oblique angles 767 from suitable directions [28] [29]. Such systems typically re768 quire multi-pass optimization, and the outputs are often plagued 769 with low contrast and ghosting or cross talk between the differ770 ent images.

Instead of structuring the geometry of the output artifact to 772 produce images through shadows, other researchers have used 773 optimization algorithms to tailor the shape of a reflector so as
774 to create a desired illumination pattern [30]. In yet another ap775 proach, transparent materials are micro-machined, and refrac776 tion is used to form the desired image as a superposition of 777 properly tailored caustics [31].

778 The emergence of multi-material RP machines [32] makes 779 it possible to create physical output with novel optical prop780 erties or varying stress-strain relationships. In the first sector ${ }_{781}$ researchers have created flat panels or 3D models from materi782 als with custom-designed subsurface-scattering properties [33] 783 [34]. This approach, which is also based on physical simulation 784 and multi-pass optimization, makes it possible to emulate the 785 look of marble, jade, or even a slice of salmon. By varying the 786 deposited materials from layer to layer, as well as adjusting the 787 porosity with which these layers are deposited, the strength and 788 pliability of the resulting composite also can be controlled and 789 finely tuned. Researchers have been able to closely model and 790 reproduce the bending and compression behavior of the soles 791 of flip-flops or slippers [35].

792 An even tougher challenge is to create complex 3D mod793 els that can change shape. Among the many different possi794 ble approaches, there are some inspiring trend-setters. Some 795 of the rapid prototyping methods allow in-situ construction of 796 interlocking assemblies of movable parts. This allows the pro797 duction of demonstration models of wrenches [36] or of com798 plete gear boxes [37]. Another approach is to create articu799 lated figures from snap-together parts, so that phantasy crea800 tures designed in Spore [38] can be brought to life as toys that 801 can assume many different poses [39]. Another highly intrigu802 ing problem is the design and fabrication of balloon hulls that, 803 when inflated to the proper size, will assume a desired shape. 804 Since such membranes do not inflate in a linear manner at all, 805 the initial limp hulls have to be given some quite different shapes. 806 Again, a multi-pass optimization step comprising detailed phys807 ical simulation can lead to success [40]. There seems to be no 808 limit to the modalities by which computer graphics design and 809 physical output can be related.

\section{10. Summary and Conclusion}

Interactive computer graphics has come a long way since ${ }_{812}$ Ivan Sutherland first demonstrated his Sketchpad [41]. Remark${ }_{813}$ able progress has been made in photo-realistic rendering, simu814 lation, immersive 3D worlds, and haptic feedback. Further de815 velopments will couple the virtual domain of computer graphics 816 and the 3D world of physical artifacts in ever more diverse and 817 intriguing ways. Most of the techniques mentioned in the pre818 vious section are at a stage of demonstrating feasibility. It will ${ }_{819}$ take some time before they turn into mainstream output tech820 nologies that can readily be applied at the push of a button.

To produce substantial and durable physical artifacts is still 822 a venture that takes special attention for most new models. I ${ }_{823}$ have learned that every time when I work on a new sculpture 824 family, or switch materials from bronze to stone, to sintered 825 steel, or to translucent polyester resins, a new set of problems ${ }_{826}$ emerge; those need careful attention and often require some ${ }_{827}$ preliminary testing of the modeling as well as of the fabrication ${ }_{828}$ technologies. The 3D Hilbert Cube (Fig.2a), made with the 
${ }_{829}$ ExOne metal-sintering process [42], required three attempts, 830 comprising an ever-increasing number of internal support rods. ${ }_{831}$ These auxiliary struts were necessary to hold this shape to832 gether in its green state as it is inserted into the sintering oven, 83 because at this stage the model has the weight of iron, but only 834 the strength of the selectively deposited binder that holds the ${ }_{835}$ stainless steel particles together.

${ }_{836}$ For many of the techniques mentioned in Section 9, mature 837 processes and services will develop over time, and will eventu838 ally allow a one-click order of the desired physical output. One 839 such example is the stone-carving service offered by Dingli in ${ }_{840}$ China [43]. All I had to do to obtain the four foot tall granite ${ }_{841}$ sculpture shown in Figure $2 \mathrm{~b}$ was to send them a six inch tall ${ }_{842} \mathrm{RP}$ model of the top part; they did not even use the CAD files ${ }_{843}$ for the actual production.

844 As discussed in Sections 7 and 8, the coupling between 845 computer graphics and the physical world is not a one-way 846 street. Pre-existing artifacts, as well as RP models with tailor847 made deformation properties, could serve as design elements 848 for inputting shapes into a CAD environment at the early stages 849 of a design process. However, both the input end, as well as the 850 output end of a computer-based design effort, require signifi851 cant advances in tool development and user-interface design to 852 make these transition points reliable and easy to use.

\section{11. Acknowledgements}

854 This work is supported in part by the National Science Foun855 dation: NSF award \#CMMI-1029662 (EDI).

\section{${ }_{856}$ References}

857 [1] MakerBots . Desktop 3d printers. web page:

858 - http://www.makerbot.com/; 2013.

859 [2] Shapeways . 3d printed products. web page:

860 - http://www.shapeways.com/; 2013.

861 [3] Ponoko. The world's easiest making system. web page:

862 - http://www.ponoko.com/; 2013.

863 [4] SecondLife. A popular virtual space. web page:

$864 \quad$ - http://maps.secondlife.com/; 2012.

865 [5] Collins B. Brent collins: Photo gallery by philip geller. web page:

$866-$ http://bridgesmathart.org/bcollins/bcollins.html; 2013.

867 [6] Francis GK, Collins B. On knot-spanning surfaces: An illustrated essay 868 on topological art. Leonardo 1992;25(3,4):313-20.

869 [7] Scherk HF. Bemerkungen über die kleinste fläche innerhalb gegebener 870 grenzen. J Reine Angew Math (Crelle's Journal) 1834;13(3,4):185-208.

871 [8] Séquin CH. Sculpture Generator I. web page:

872 - http://www.cs.berkeley.edu/ sequin/GEN/; 2013.

873 [9] Séquin $\mathrm{CH}$. Virtual prototyping of scherk-collins saddle rings. Leonardo $874 \quad 1997 ; 30(2): 89-96$.

875 [10] Reinmuth S. Reinmuth bronze studio. web page:

876 - http://www.reinmuth.com/; 2013.

877 [11] Smith J. Slide design environment. web page:

878 - http://www.cs.berkeley.edu/ ug/slide/docs/slide/spec/; 2004

879 [12] Scan\&Solve . Scan-and-solve for rhino. web page:

${ }_{880}-$ http://www.scan-and-solve.com; 2013.

881 [13] Lynn D. Nova blue studio arts. web page:

882 - http://sites.google.com/site/novabluestudioarts/home; 2013.

883 [14] Séquin CH. Whirled White Web. web page:

884 - http://www.cs.berkeley.edu/ sequin/SCULPTS/SnowSculpt03/; 2013.

885 [15] SolidWorks . 3d design simplified. web page:

$886-$ http://www.solidworks.com/; 2013.

887 [16] Stratasys . For a 3d world. web page: - http://www.stratasys.com/; 2013.
888 [17] Andrews J, Jin H, Séquin CH. Interactive inverse 3d modeling. ComputerAided Design and Applications 2012;9.

[18] Pottmann H, Randrup T. Generalized, basis-independent kinematic surface fitting. Computing 1998;60:307-22.

2 [19] Andrews J, Séquin CH. Generalized, basis-independent kinematic surface fitting. Computer-Aided Design 2013;45(3):615-20.

4 [20] Dachille F, Qin H, Kaufman A. A novel haptics-based interface and sculpting system for physics-based geometric design. Computer-Aided Design 2001;33(5):403-20.

[21] Shon Y, McMains S. Evaluation of drawing on 3d surfaces with haptics. IEEE Computer Graphics and Applications 2004;24(6):40-50.

9 [22] Gehry F. The architecture of frank gehry. web page: - http://www.gehrytechnologies.com/architecture/recent-work; 2012.

1 [23] Zhang L, Curless B, Seitz SM. Rapid shape acquisition using color structured light and multi-pass dynamic programming. In: The 1st IEEE International Symposium on 3D Data Processing, Visualization, and Transmission. 2002, p. 24-36.

5 [24] Xbox . Introducing kinect. web page: - http://www.xbox.com/en-US/KINECT; 2013.

7 [25] MAKEeditors . Ultimate guide to 3d printing. Make 2012;:1-116.

8 [26] Weyrich T, Deng J, Barnes C, Rusinkiewicz S, Finkelstein A. Digital bas-relief from 3d scenes. ACM Trans Graph 2007;26(3):3201-7.

0 [27] DiCarlo J, Wandell B. Rendering high dynamic range images. Proc SPIE Electronic Imaging Conf 2000;3965:392-401.

912 [28] Alexa M, Matusik W. Images from self-occlusion. Computational Aesthetics in Graphics, Visualization, and Imaging (CAe2011) 2011;:17-24.

914 [29] Alexa M, Matusik W. Reliefs as images. ACM Transactions on Graphics (SIGGRAPH'10) 2010;29(4):6001-7.

916 [30] Patow G, Pueyo X, Vinacua A. User-guided inverse reflector design. Computers \& Graphics 2007;31:501-15.

918 [31] Papas M, Jarosz W, Jakob W, Rusinkiewicz S, Matusik W, Weyrich

919 T. Goal-based caustics. Computer Graphics Forum (Eurographics'11) $920 \quad 2011 ; 30(2): 503-11$

921 [32] Stratasys . Objetconnex, multi-material 3d printing system. web page: - http://objet.com/3d-printers/connex/objet-connex500/; 2013.

923 [33] Hasan M, Fuchs M, Matusik W, Pfister H, Rusinkiewicz S. Physical reproduction of materials with specified subsurface scattering. ACM Transactions on Graphics (SIGGRAPH'10) 2010;29(4):6101-10.

26 [34] Dong Y, Wang J, Pellacini F, Tong X, Guo B. Fabricating spatiallyvarying subsurface scattering. ACM Transactions on Graphics (SIGGRAPH'10) 2010;29(4):6201-10.

[35] Bickel B, Bächer M, Otaduy M, Lee H, Pfister H, Gross M, et al. Design and fabrication of materials with desired deformation behavior. ACM Transactions on Graphics (SIGGRAPH'10) 2010;29(3):6301-10.

2 [36] Custom3dSolutions. Your source for rapid prototyping and $3 \mathrm{~d}$ cad services. web page: - http://custom3dsolutions.com/; 2013.

35 [37] PRGprototyping. Bringing ideas to reality. web page: - http://prgprototyping.com/about.htm; 2013.

937 [38] Spore. How will you create the universe? web page: - http://www.spore.com/; 2013.

[39] Moritz Bächer M, Bickel B, James D, Pfister H. Fabricating articulated characters from skinned meshes. ACM Transactions on Graphics (SIGGRAPH'12) 2012;31(4):4701-9.

42 [40] Skouras M, Thomaszewski B, Bickel B, Gross1 M. Computational design of rubber balloons. Computer Graphics Forum (Eurographics'12) 2012;31:835-44.

945 [41] Sutherland IE. SketchPad: A man-machine graphical communication system. In: AFIPS Conference Proceedings; vol. 23. 1963, p. 323-8.

947 [42] ExOne . Digital part materialization. web page: - http://www.exone.com/; 2013.

949 [43] Dingli . Fujian dingli stone carving art co., ltd. web page: 